\title{
Ranking business intelligence factors influencing on development of export
}

\author{
Hamideh Lotfi* and Abdollah Naami
}

Department of Management and Accounting, South Tehran Branch, Islamic Azad University, Tehran, Iran

\begin{tabular}{l}
\hline C H R O N I C L E \\
\hline Article history: \\
Received December 18, 2014 \\
Accepted March 262015 \\
Available online \\
April 112015 \\
\hline Keywords: \\
Business intelligence \\
Competitive position \\
Organizational resources \\
Foreign trade \\
\hline
\end{tabular}
\begin{abstract}
A B S T R A C T
This paper presents an empirical investigation to determine important business intelligence factors influencing on development of export activities. The study selects a sample of business developers who were involved in export activities in city of Tehran, Iran. Cronbach alpha based on standardized items was calculated as 0.882 , which is well above the minimum desirable level. In addition, Bartlett's test of Sphericity yields a Chi-Square value of 3242.82 ( $\mathrm{df}=861$, Sig. $=0.000)$. Using principle component analysis, the study has determined four factors including competitive position, organizational resources, efficient system and customer orientation influencing on development of export activities.
\end{abstract}

(c) 2015 Growing Science Ltd. All rights reserved.

\section{Introduction}

Export has been considered as one of the most important activities for business development in developing countries (Rahchamandi \& Fallahi, 2014). There are literally several studies on factors influencing on export activities (Turban et al., 2007; Tsoukiàs, 2008). Suárez-Ortega and Alamo-Vera (2005), for instance, studied the specific organizational and managerial determinants of different characteristics of firms' export development process including intention, propensity, and intensity. They reported that factors influencing on export involvement were not the same along the process of export development. Atuahene-Gima (1995) studied the role of new product factors in the firm's propensity to export and its performance in exporting new products based on a sample of Australian companies. They stated that product advantage, proficiency of predevelopment activities, and international orientation of the development process had positive effects on firm's propensity to export new products. Besides, the new product's domestic market performance and its impact on the sales and profitability of other products of the company were substantially associated with its export performance. Leonidou et al. (2007) presented an analytical review of the factors stimulating smaller firms to export. Business intelligence is also another important factor for development of organizational strategies (Alnoukari, 2009). There are several methods for determining business intelligence in organizations such as data mining (Carlo, 2009) and rule-induction framework (Chung \& Tseng, 2012). Elbashir et al. (2008) measured the effects of business intelligence systems by investigating the 
relationship between business process and organizational performance. Herschel and Jones (2005) investigated the relationship between knowledge management and business intelligence. Olszak and Ziemba (2007) presented an approach for building and implementing business intelligence systems.

\section{The proposed method}

This paper presents an empirical investigation to determine important business intelligence factors influencing on development of export activities. The study selects a sample of business developers who were involved in export activities in city of Tehran, Iran.

\section{Table 1}

The summary of questions of the survey

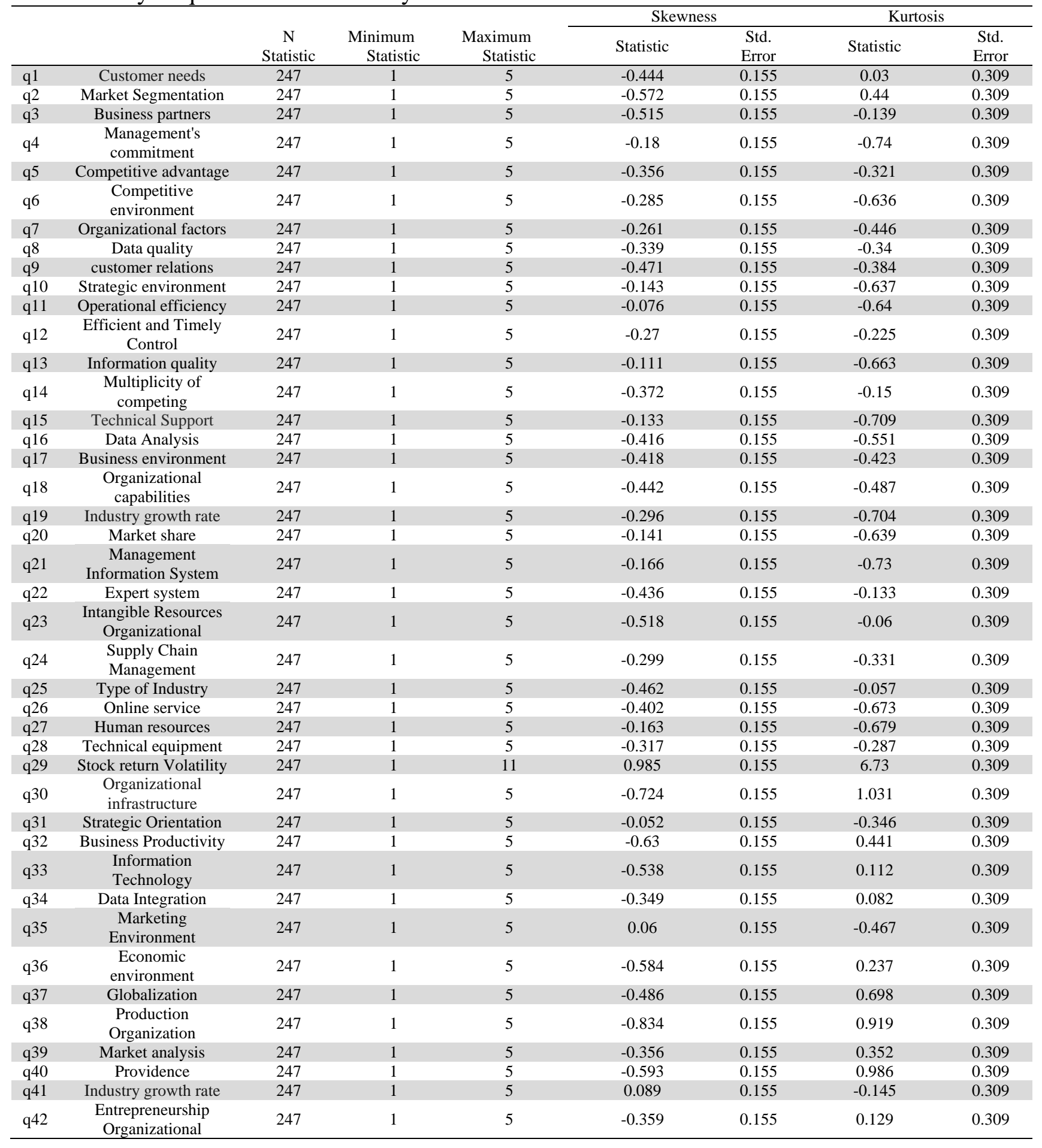


Cronbach alpha based on standardized items was calculated as 0.882 , which is well above the minimum desirable level. In addition, Bartlett's test of Sphericity yields a Chi-Square value of 3242.82 ( $\mathrm{df}=861$, Sig. $=0.000$ ). Table 1 shows some basic statistics associated with different questions of the survey. As we can observe from the results of Table 1, some components of the survey are not within desirable limits and we may, therefore, use principal component analysis (PCA) after eliminating six items. Table 2 shows the results of PCA before rotation. In addition, Fig. 1 shows details of Scree plot.

Table 2

The summary of PCA before rotation

\begin{tabular}{|c|c|c|c|c|c|c|c|c|c|}
\hline \multirow[b]{2}{*}{ Component } & \multicolumn{3}{|c|}{ Initial Eigen values } & \multicolumn{3}{|c|}{ Extraction Sums of Squared Loadings } & \multicolumn{3}{|c|}{ Rotation Sums of Squared Loadings } \\
\hline & Total & $\begin{array}{c}\% \text { of } \\
\text { Variance }\end{array}$ & $\begin{array}{c}\text { Cumulative } \\
\% \\
\end{array}$ & Total & $\begin{array}{c}\text { \% of } \\
\text { Variance }\end{array}$ & $\begin{array}{c}\text { Cumulative } \\
\% \\
\end{array}$ & Total & $\begin{array}{c}\% \text { of } \\
\text { Variance }\end{array}$ & $\begin{array}{c}\text { Cumulative } \\
\% \\
\end{array}$ \\
\hline 1 & 7.784 & 21.623 & 21.623 & 7.784 & 21.623 & 21.623 & 3.246 & 9.016 & 9.016 \\
\hline 2 & 3.063 & 8.509 & 30.131 & 3.063 & 8.509 & 30.131 & 2.767 & 7.686 & 16.702 \\
\hline 3 & 1.913 & 5.315 & 35.446 & 1.913 & 5.315 & 35.446 & 2.441 & 6.781 & 23.483 \\
\hline 4 & 1.503 & 4.174 & 39.62 & 1.503 & 4.174 & 39.62 & 2.313 & 6.425 & 29.908 \\
\hline 5 & 1.327 & 3.687 & 43.307 & 1.327 & 3.687 & 43.307 & 2.248 & 6.243 & 36.152 \\
\hline 6 & 1.298 & 3.605 & 46.912 & 1.298 & 3.605 & 46.912 & 2.019 & 5.61 & 41.761 \\
\hline 7 & 1.254 & 3.484 & 50.396 & 1.254 & 3.484 & 50.396 & 1.681 & 4.669 & 46.43 \\
\hline 8 & 1.158 & 3.218 & 53.614 & 1.158 & 3.218 & 53.614 & 1.577 & 4.381 & 50.811 \\
\hline 9 & 1.088 & 3.021 & 56.635 & 1.088 & 3.021 & 56.635 & 1.476 & 4.1 & 54.911 \\
\hline 10 & 1.055 & 2.93 & 59.565 & 1.055 & 2.93 & 59.565 & 1.375 & 3.818 & 58.729 \\
\hline 11 & 1.02 & 2.834 & 62.399 & 1.02 & 2.834 & 62.399 & 1.321 & 3.67 & 62.399 \\
\hline 12 & 0.903 & 2.507 & 64.906 & & & & & & \\
\hline 13 & 0.872 & 2.421 & 67.327 & & & & & & \\
\hline 14 & 0.837 & 2.325 & 69.652 & & & & & & \\
\hline 15 & 0.79 & 2.195 & 71.847 & & & & & & \\
\hline 16 & 0.76 & 2.112 & 73.959 & & & & & & \\
\hline 17 & 0.724 & 2.01 & 75.969 & & & & & & \\
\hline 18 & 0.709 & 1.971 & 77.939 & & & & & & \\
\hline 19 & 0.646 & 1.794 & 79.734 & & & & & & \\
\hline 20 & 0.631 & 1.753 & 81.487 & & & & & & \\
\hline 21 & 0.615 & 1.71 & 83.197 & & & & & & \\
\hline 22 & 0.597 & 1.658 & 84.854 & & & & & & \\
\hline 23 & 0.539 & 1.496 & 86.351 & & & & & & \\
\hline 24 & 0.508 & 1.411 & 87.762 & & & & & & \\
\hline 25 & 0.496 & 1.378 & 89.141 & & & & & & \\
\hline 26 & 0.473 & 1.315 & 90.456 & & & & & & \\
\hline 27 & 0.435 & 1.208 & 91.664 & & & & & & \\
\hline 28 & 0.432 & 1.199 & 92.863 & & & & & & \\
\hline 29 & 0.397 & 1.103 & 93.965 & & & & & & \\
\hline 30 & 0.388 & 1.077 & 95.043 & & & & & & \\
\hline 31 & 0.361 & 1.004 & 96.047 & & & & & & \\
\hline 32 & 0.342 & 0.949 & 96.996 & & & & & & \\
\hline 33 & 0.307 & 0.854 & 97.85 & & & & & & \\
\hline 34 & 0.292 & 0.812 & 98.662 & & & & & & \\
\hline 35 & 0.255 & 0.709 & 99.371 & & & & & & \\
\hline 36 & 0.226 & 0.629 & 100 & & & & & & \\
\hline
\end{tabular}

Scree Plot

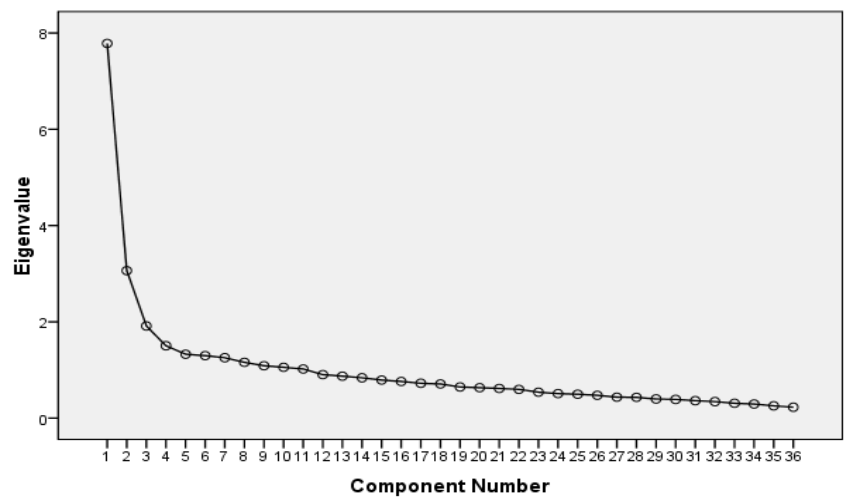

Fig. 1. The summary of Scree plot 
According to the results of Table 2 and Fig. 1, we may understand that there were four factors influencing on development of export activities. Table 3 presents the results of PCA after rotation.

Table 3

The summary of PCA after rotation

\begin{tabular}{|c|c|c|c|c|c|c|c|c|c|c|c|c|}
\hline & \multirow{2}{*}{ Factor } & \multicolumn{11}{|c|}{ Rotated Component Matrix ${ }^{\mathrm{a}}$} \\
\hline & & 1 & 2 & 3 & 4 & 5 & 6 & 7 & 8 & 9 & 10 & 11 \\
\hline q16 & Data Analysis & 0.652 & & & & & & & & & & \\
\hline q22 & EXPERT SISTEM & 0.618 & & & & & & & & & & \\
\hline q15 & Technical Support & 0.566 & 0.402 & & & & & & & & & \\
\hline q8 & Data quality & 0.496 & & & & & & & & & & \\
\hline q12 & $\begin{array}{l}\text { Efficient and Timely } \\
\text { Control }\end{array}$ & 0.48 & & & & 0.416 & & & & & & \\
\hline q21 & MIS & 0.445 & & & & & 0.394 & & & 0.393 & & \\
\hline q13 & Information quality & 0.404 & 0.346 & & & & & & & & & \\
\hline q17 & Business environment & & 0.7 & & & & & & & & & \\
\hline q20 & Market share & 0.379 & 0.594 & & & & & & & & & \\
\hline q19 & Industry growth rate & & 0.559 & & & & 0.41 & & & & & \\
\hline q14 & Multiplicity of competing & & 0.527 & & & 0.432 & & & & & & \\
\hline q25 & Type of Industry & & 0.47 & 0.379 & & & & & & & & \\
\hline q5 & Competitive advantage & & 0.464 & & & 0.399 & & & & & & \\
\hline q28 & Technical equipment & & & 0.754 & & & & & & & & \\
\hline q27 & Human resources & & & 0.693 & & & & & & & & \\
\hline q23 & Intangible Resources Organization & & & 0.488 & & & & & & 0.418 & & \\
\hline q4 & Management's commitment & 0.445 & & 0.456 & & & & & & & & \\
\hline q18 & Organizational capabilities & & 0.334 & 0.395 & & & & & & -0.372 & & \\
\hline q37 & Globalization & & & & 0.736 & & & & & & & \\
\hline q40 & Providence & & & & 0.724 & & & & & & & \\
\hline q39 & Market analysis & & & & 0.677 & & & & & & & \\
\hline q36 & Economic environment & & & & 0.4 & & -0.379 & 0.387 & & & & 0.396 \\
\hline q10 & Strategic environment & & & & & 0.752 & & & & & & \\
\hline q11 & Operational efficiency & & & & & 0.736 & & & & & & \\
\hline q9 & customer relations & & & & & & 0.699 & & & & & \\
\hline q26 & Online service & 0.443 & & & & & 0.464 & & & & & \\
\hline q1 & customer needs & & & & & & 0.441 & 0.361 & & & & \\
\hline q2 & Market Segmentation & & & & & & 0.39 & & & & & \\
\hline q7 & Organizational factors & & & & & & & 0.666 & & & & \\
\hline q41 & Industry growth rate & & & & & & & & 0.806 & & & \\
\hline q31 & Strategic Orientation & & & 0.389 & & & & & 0.514 & & & \\
\hline q42 & Entrepreneurship Organizational & & & & & & & & 0.463 & 0.413 & & \\
\hline q35 & Marketing Environment & & & & 0.362 & & & & & 0.676 & & \\
\hline q34 & Data Integration & & & & & & & & & & 0.818 & \\
\hline q32 & Business Productivity & & & & & & & 0.455 & & & 0.47 & \\
\hline q30 & Organizational infrastructure & & & & & & & & & & & 0.776 \\
\hline
\end{tabular}

\section{Discussion and conclusion}

Based on the implementation of PCA method, the study has determined four factors including competitive position, organizational resources, efficient system and customer orientation influencing on development of export activities. In our survey, customer orientation $(r=0.985$, Sig. $=0.000)$ has been the most important factor followed by organizational resources $(r=0.949$, Sig. $=0.000)$, efficient system $(r=0.919$, Sig. $=0.000)$ and competitive position $(r=0.836$, Sig. $=0.000)$. 
The results of this survey are somewhat consistent with similar studies accomplished earlier. For instance, Yazdi et al. (2014) presented a study to detect important factors influencing exporting herbal supplements and determined eight factors including supportive laws and regulations, organizational atmosphere, marketing structure, knowledge oriented, feasibility study, research and development, competitive strategy and partnership strategies. Nikseresht (2013) considered whether or not improving relationships between countries could positively impact on empowering firms and export capabilities. He also considered whether or not improving national strategies for developing exports could positively impact on empowering companies and export capabilities. He also tried to determine whether or not changes on rules and regulations could harm export capabilities. He reported that empowering small and mid-cap firms could contribute the whole economy through boosting export.

Azad and Savadkouhi (2013) also presented an empirical study on factors influencing on insurance issued by export guarantee funds. They determined four factors including risk management, customer oriented, quality management and trade management. Finally, Rahchamandi and Fallahi (2014) presented a study on logistics outsourcing on exports of minerals goods. They determined that there had been a meaningful relationship between strategic orientations of exporters against outsourcing third part logistics (3PL) and basic and additional capabilities of 3PL. Babakhani and Haji (2011) performed a study to determine the most important obstacles on boosting exporting industry in one of the provinces of Iran. According to the results of this study, the government could help 28 producers develop their exporting business by reducing tax, providing low interest loans, supporting marketing planning abroad, etc. There were also different parameters which could be considered by producers such as having an active union, maintaining a high level of quality for long term, using a good packaging, etc.

\section{Acknowledgement}

The authors would like to thank the anonymous referees for constructive comments on earlier version of this paper.

\section{References}

Alnoukari, M. (2009). Using business intelligence solutions for achieving organization's strategy: Arab international university case study. Internetworking Indonesia Journal, 1(2), 11-16.

Atuahene-Gima, K. (1995). The influence of new product factors on export propensity and performance: an empirical analysis. Journal of International Marketing, 3(2), 11-28.

Azad, N \& Savadkouhi, R. (2013). An empirical investigation on factors influencing on insurance issued by export guarantee funds. Management Science Letters, 3(7), 2101-2106.

Babakhani, M \& Haji, H. (2011). An empirical study to determine the critical success factors of export industry. Management Science Letters, 1(1), 23-28.

Carlo, V. (2009). Business intelligence: data mining and optimization for decision making. Editorial John Wiley and Sons.

Chung, W., \& Tseng, T. L. B. (2012). Discovering business intelligence from online product reviews: A rule-induction framework. Expert systems with applications, 39(15), 11870-11879.

Elbashir, M. Z., Collier, P. A., \& Davern, M. J. (2008). Measuring the effects of business intelligence systems: The relationship between business process and organizational performance. International Journal of Accounting Information Systems, 9(3), 135-153.

Herschel, R. T., \& Jones, N. E. (2005). Knowledge management and business intelligence: the importance of integration. Journal of Knowledge Management,9(4), 45-55.

Leonidou, L. C., Katsikeas, C. S., Palihawadana, D., \& Spyropoulou, S. (2007). An analytical review of the factors stimulating smaller firms to export: Implications for policy-makers. International Marketing Review, 24(6), 735-770.

Nikseresht, F. (2013). How empowering small and mid-cap firms develops national exports. Management Science Letters, 3(10), 2661-2664. 
Olszak, C. M., \& Ziemba, E. (2007). Approach to building and implementing business intelligence systems. Interdisciplinary Journal of Information, Knowledge, and Management, 2(2007), 134-148.

Rahchamandi, E \& Fallahi, K. (2014). An investigation on logistics outsourcing on exports of minerals goods. Uncertain Supply Chain Management, 2(3), 163-166.

Suárez-Ortega, S. M., \& Alamo-Vera, F. R. (2005). SMES' internationalization: firms and managerial factors. International Journal of Entrepreneurial Behaviour \& Research, 11(4), 258-279.

Tsoukiàs, A. (2008). From decision theory to decision aiding methodology. European Journal of Operational Research, 187(1), 138-161.

Turban, E., Sharda, R., Delen, D., \& Efraim, T. (2007). Decision support and business intelligence systems. Pearson Education India.

Yazdi, N., Naami, A \& Azad, N. (2014). An empirical investigation on factors influencing export of herbal supplements. Management Science Letters, 4(2), 347-352. 